Article

\title{
Some Results in Green-Lindsay Thermoelasticity of Bodies with Dipolar Structure
}

\author{
Marin Marin ${ }^{1,+}+\mathbb{D}$, Eduard M. Craciun ${ }^{2, *,+}$ and Nicolae Pop ${ }^{3,+}$ \\ 1 Department of Mathematics and Computer Science, Transilvania University of Brasov, 500036 Brasov, Romania; \\ m.marin@unitbv.ro \\ 2 Faculty of Mechanical, Industrial and Maritime Engineering, Ovidius University of Constanta, \\ 900527 Constanta, Romania \\ 3 Institute of Solid Mechanics of Romanian Academy, 010141 Bucharest, Romania; nicpop@gmail.com \\ * Correspondence: mcraciun@univ-ovidius.ro \\ + These authors contributed equally to this work.
}

Received: 16 March 2020; Accepted: 29 March 2020; Published: 2 April 2020

check for updates

\begin{abstract}
The main concern of this study is an extension of some results, proposed by Green and Lindsay in the classical theory of elasticity, in order to cover the theory of thermoelasticity for dipolar bodies. For dynamical mixed problem we prove a reciprocal theorem, in the general case of an anisotropic thermoelastic body. Furthermore, in this general context we have proven a result regarding the uniqueness of the solution of the mixed problem in the dynamical case. We must emphasize that these fundamental results are obtained under conditions that are not very restrictive.
\end{abstract}

Keywords: thermoelasticity; dipolar bodies; Green-Lindsay theory; reciprocal result; uniqueness

MSC: 73B30; 73C35; 73C60

\section{Introduction}

As it is known, some generalized thermoelastic models have been proposed for transient responses in many applications, like ultra-fast lasers heating, or low temperatures; that is, in situations where classical thermoelasticity fails. Some representative theories in this regard can be found in [1-7]. Furthermore, in books $[8,9]$ we find some models for generalized thermoelastic media. The thermoelastic model proposed by Green and Lindsay in [1] is one of such theory, and it takes the second sound effect of heat conduction into account.

We want to say that we dedicated our study to this theory because it has aroused much interest in recent years. Unlike the classical thermoelasticity theories, in the Green and Lindsay thermoelasticity theory, among the constitutive variables, it is included the temperature rate. As a consequence, in this theory it is anticipated that the heat waves propagate at finite speed.

Since waves propagating at concrete finite speed were observed in the case of all solids, it is natural to consider that this theory is physically more realistic and more general than the classical theory. That is why we can observe a rapidly increasing interest of many works for this theory, and in which we can find several problems revealing interesting phenomena which characterize it. Another generalization of our study concerns the intimate structure of the body. We approach a thermoelastic dipolar body, knowing that the dipolar structure appears as a result of the inclusion, in the theory, of the idea of the unit cell. A solid with dipolar structure can model a grain of a granular material, a crystallite of a polycrystalline, 
a molecule of a polymer, etc. We can emphasize the importance of the dipolar materials, if we analyze the importance given to this structure by some of the known researchers. Among the works elaborated for this purpose, it is considered that the papers of Mindlin [10], of Green and Rivlin [11], and the book Gurtin et al. [12] are very relevant from this regards. Other aspects of the bodies with dipolar structure are addressed in the studies [13-28]. In [14], the authors introduced the coupled theory, the Lord-Schulman theory with one relaxation time and the Green-Lindsay theory with two relaxation times to study the influence of the magnetic field and rotation on the 2-D problem of a fiber-reinforced thermoelastic body and the interaction with each other under the influence of gravity. Furthermore, in [15] the authors have shown that the double porosity structure of the body is influenced by the displacement field, which is consistent with real models. The main result of the paper [17] describes a class of semi-inverse solutions to the Saint-Venant's problem in terms of some generalized plane strain problems. In [18] it can be found a result regarding the temporal behavior of our thermoelastic body with a dipolar structure, which is studied by means of some relations on a partition of various parts of the energy associated to the solution of the problem. The system of differential equations considered in [22] is solved numerically and some plots for displacement, radial and electromagnetic stresses, and temperature are presented. In [27] the authors study qualitative properties of the solutions of the system of partial differential equations modeling thermomechanical deformations for mixtures of thermoelastic solids when the theory of Green and Lindsay for the heat conduction is considered.

It should be emphasized that Green and Lindsay's theory has been addressed for many types of media: classical thermoelastic environments [1], dynamic thermoelasticity [29], mixture of Green-Lindsay thermoelastic solids [30], thermoviscoelastic environments [31] and so on. In our work we use this theory in the context of the dipolar thermoelastic bodies.

The structure of our work is as follows. The mixed problem for Green-Lindsay thermoelasticity of bodies with dipolar structure is defined in Section 2. Then, we introduce the basic notations, the motion equations and the energy equation, the initial data and the boundary conditions. In the last section we present the main results. First, we establish a reciprocity relation which involves thermoelastic processes at different instants. Then we show that this relation can be used to obtain uniqueness theorems. Both reciprocity theorem and also the uniqueness theorems are derived avoiding the use of the definiteness assumption on the elasticity tensors. Furthermore, we avoided to impose the hypothesis that the tensor of conductivity $k_{i j}$ is a positive definite one.

\section{Notations and Basic Equations}

Consider that our thermoelastic dipolar solid occupies at the initial time $t=0$ a region $B$ of the space $R^{3}$ which is assumed to be properly regular.

We denote by $\partial B$ the border of the region $B$ and suppose that the surface $\partial B$ has the regularity necessary to be able to apply the divergence theorem. Furthermore, we will use the notation $\bar{B}=B \cup \partial B$, where $\bar{B}$ is called the closure of the domain $B$. The unit normal of the boundary $\partial B$ has the components $n_{i}$ and is oriented towards the outside of the surface.

The evolution of our medium is reported to a system of Cartesian axes $O x_{i}(i=1,2,3)$. The Cartesian vector and tensor notation is adopted. The temporal variable is $t, t \in[0, \infty)$, and the notation $x=\left(x_{i}\right)$ is used for any point in $B$.

The Greek indices take only the values 1,2, whereas for the Italic indices we always assume the values $1,2,3$. A superposed dot stands for the material derivative with respect to time variable. A subscript after a comma, $j$, denotes the partial derivative with respect to coordinate $x_{j}$. We will use the Einstein summation convention with respect to the summation over indices that repeat. When there is no risk of confusion, we will refrain from specifying the temporal or spatial argument of a function. 
The independent variables which describe the evolution of a dipolar body in the context of the Green-Lindsay thermoelasticity will be described with the help of the following variables:

- $u_{i}=u_{i}(x, t)$ the components of displacement;

- $\phi_{j k}=\phi_{j k}(x, t)$ the components of dipolar displacement;

- $\theta=\theta(x, t)$ the temperature.

As measures of the deformation, we will use the strain tensors $e_{i j}, v_{i j}$ and $\chi_{i j k}$ which are introduced by using the following geometric equations:

$$
e_{i j}=\frac{1}{2}\left(u_{j, i}+u_{i, j}\right), \quad v_{i j}=u_{j, i}-\phi_{i j}, \chi_{i j k}=\phi_{i j, k} .
$$

Our next analyzes are made within a linear theory. As a consequence, we will expand the free energy function $W$ in MacLaurin series and retain only the linear and quadratic terms. If we take into account that the initial state (the reference state) is supposed free of charges, we are led to the conclusion that the energy function has the following expression:

$$
\begin{aligned}
& W= \frac{1}{2} A_{i j m n} e_{i j} e_{m n}+D_{i j m n} e_{i j} v_{m n}+F_{i j m n r} e_{i j} \chi_{m n r}+ \\
&+\frac{1}{2} B_{i j m n} v_{i j} v_{m n}+G_{i j m n r} v_{i j} \chi_{m n r}+\frac{1}{2} C_{i j k m n r} \chi_{i j k} \chi_{m n r}+ \\
&+\left(a_{i j} e_{i j}+b_{i j} v_{i j}+c_{i j k} \chi_{i j k}\right)(\theta+\alpha \dot{\theta})-\frac{1}{2} d(\theta+\alpha \dot{\theta})^{2}+\frac{1}{2} k_{i j} \theta_{, i} \theta_{, j} .
\end{aligned}
$$

In what follows we will use the notations $t_{i j}, \sigma_{i j}$ and $\eta_{i j k}$ for the tensors of stress.

By using a similar procedure to the one that was handled by Coleman and Noll (see [32]) so that if we substitute the free energy in the entropy production inequality, we are led to a relation from where we obtain:

- the motion equations:

$$
\begin{aligned}
& \left(t_{i j}+\sigma_{i j}\right)_{, j}+\varrho f_{i}=\varrho \ddot{u}_{i}, \\
& \eta_{i j k, i}+\sigma_{j k}+\varrho g_{j k}=I_{j r} \ddot{\phi}_{k r} ;
\end{aligned}
$$

- the equation of energy:

$$
\rho T_{0} \dot{S}=q_{i, i}+r
$$

Furthermore, by using the procedure of Green and Rivlin from [11], we obtain thermodynamics restrictions on constitutive functions. As it is known the constitutive equations give the expression of stress tensors as functions of the strain tensors and some constants of the material:

$$
\begin{gathered}
t_{i j}=\frac{\partial W}{\partial e_{i j}}=A_{i j m n} e_{m n}+D_{m n i j} v_{m n}+F_{m n r i j} \chi_{m n r}+a_{i j}(\theta+\alpha \dot{\theta}), \\
\sigma_{i j}=\frac{\partial W}{\partial v_{i j}}=D_{i j m n} e_{m n}+B_{i j m n} v_{m n}+G_{i j m n r} \chi_{m n r}+b_{i j}(\theta+\alpha \dot{\theta}), \\
\eta_{i j k}=\frac{\partial W}{\partial \chi_{i j k}}=F_{i j k m n} e_{m n}+G_{m n i j k} v_{m n}+C_{i j k m n r} \chi_{m n r}+c_{i j k}(\theta+\alpha \dot{\theta}), \\
S=-\frac{\partial W}{\partial(\theta+\alpha \dot{\theta})}=-a_{i j} e_{i j}-b_{i j} v_{i j}-c_{i j k} \chi_{i j k}+d(\theta+\alpha \dot{\theta}) .
\end{gathered}
$$


It is known that the internal energy of the elastic media (let us denote it by $e$ ) depends on the deformation tensors. In the case of a thermoelastic media it is added the temperature as an independent variable and its derivative, as such it is introduced the free energy, which is called Helmholtz, denoted by $W$, which takes into account the temperature $\theta$ and the entropy $S$, namely $W=e-\theta S$. According to Green-Lindsay theory, $\theta$ and $\dot{\theta}$ are coupled as in Formula (2). In the inequality of entropy appear terms in the following form $\left(t_{i j}-\partial W / \partial e_{i j}\right), \ldots,(S-\partial W / \partial(\theta+\alpha \dot{\theta}))$, and the parentheses must be null and then we obtain the formulas in (5).

According to [1], the heat flux vector has the components expressed with the help of temperature $\theta$ through the equation:

$$
q_{i}=-\left(b_{i} \dot{\theta}+k_{i j} \theta_{, j}\right) .
$$

In view of (5) ${ }_{4}$ and (6), the energy Equation (4) receives the following form

$$
\frac{\varrho}{T_{0}} r+k_{i j} \theta_{, i j}-d \dot{\theta}-h \ddot{\theta}+a_{i j} \dot{e}_{i j}+b_{i j} \dot{v}_{i j}+c_{i j k} \dot{\chi}_{i j k}+2 b_{i} \dot{\theta}_{, i}=0 .
$$

The above equations are considered on the domain $B$ and we have used the following notations:

- $\varrho$ - the mass density in the initial state;

- $I_{j k}$-the tensor of inertia;

$-t_{i j}, \sigma_{i j}, \eta_{i j}$ - the components of the stress tensors;

- $f_{i}$ - the components of body force per unit mass;

- $g_{j k}$ - the components of dipolar body force per unit mass;

- $r$-the heat supply per unit mass;

- $S$ - the entropy per unit mass;

- $d$ - the thermal capacity;

- $q_{i}$ - the components of the heat flux vector;

- $k_{i j}$ - the thermal conductivity tensor.

Lastly, the tensors $A_{i j m n}, \ldots D_{i j m}, \ldots, a_{i j}, \ldots$ and the scalar coefficient $m$ represent the characteristic functions of the material (the constitutive coefficients) and they obey to the following symmetry relations

$$
\begin{gathered}
A_{i j m n}=A_{m n i j}=A_{i j n m}, B_{i j m n}=B_{m n i j}, D_{i j m n}=D_{j i m n}, \\
F_{i j k m n}=F_{j i k m n}, C_{i j k m n r}=C_{m n r i j k}, a_{i j}=a_{j i}, k_{i j}=k_{j i} .
\end{gathered}
$$

Now we define the surface force tractions $t_{i}$ and $\eta_{j k}$, corresponding to $u_{i}$ and $\phi_{j k}$, and, also, the heat flux $q$, which corresponds to $q_{i}$ :

$$
t_{i} \equiv\left(t_{i j}+\sigma_{i j}\right) n_{j}, \eta_{j k} \equiv \eta_{i j k} n_{i}, q \equiv q_{i} n_{i}
$$

The mixed problem in our context will be complete, if we will accompany the system of Equations (1)-(5) by the boundary conditions that follow:

$$
\begin{aligned}
& u_{i}=u_{0 i} \text { on } S_{1}, \phi_{j k}=\phi_{0 j k} \text { on } S_{2}, \theta=\theta_{0} \text { on } S_{3}, \\
& t_{i}=t_{0 i} \text { on } S_{1}^{c}, \eta_{j k}=\eta_{0 j k} \text { on } S_{2}^{c}, q=q_{0} \text { on } S_{3}^{c},
\end{aligned}
$$

where the functions $u_{0 i}, \phi_{0 j k}, \theta_{0}, t_{0 i}, \eta_{0 j k}$ and $q_{0}$ are prescribed. 
Furthermore, in (10) the surfaces $S_{1}, S_{2}$ and $S_{3}$, with their respective complements $S_{1}^{c}, S_{2}^{c}$ and $S_{3}^{c}$, are subsurfaces of the border $\partial B$ and have the following properties:

$$
\begin{gathered}
S_{1} \cup S_{1}^{c}=S_{2} \cup S_{2}^{c}=S_{3} \cup S_{3}^{c}=\partial B, \\
S_{1} \cap S_{1}^{c}=S_{2} \cap S_{2}^{c}=S_{3} \cap S_{3}^{c}=\varnothing .
\end{gathered}
$$

Regarding the initial conditions, we suppose that at initial time $(t=0)$, the medium is at rest. Furthermore, for $t=0$ the body has a null initial temperature and the temperature rate is also null. As such, we assume the following homogeneous initial conditions:

$$
u_{i}(x, 0)=\dot{u}_{i}(x, 0)=0, \phi_{j k}(x, 0)=\dot{\phi}_{j k}(x, 0)=0, \theta(x, 0)=\dot{\theta}(x, 0)=0 .
$$

Let us denote by $\mathcal{P}$ the mixed initial-boundary value problem in the context of thermoelasticity of dipolar bodies. It consists of the system of field Equations (1), (3)-(5), the boundary conditions (10) and the initial conditions (11) .

Now we systematize the restrictions that we have to impose on the functions we work with in order to be able to obtain the results we have proposed. In the following considerations, we will assume the following hypotheses of regularity:

- $u_{i}, \phi_{j k}, \theta \in C^{1}(\bar{B}) \cap C^{2}(B)$;

- the constitutive coefficients are functions of class $C^{1}(B)$;

- the supply loads $f_{i}, g_{j k}, r$ are continuous functions, i.e., of class $C^{0}(B)$;

- $e_{i j}, v_{i j}, \chi_{i j k} \in C^{1}(B) \cap C^{0}(\bar{B})$;

- $t_{i j}, \sigma_{i j}, \eta_{i j k}, q_{i} \in C^{1}(B) \cap C^{0}(\bar{B})$.

A solution $\left(u_{i}, \phi_{j k}, \theta\right)$ of the problem $\mathcal{P}$ is the effect of a system of external actions defined by: $\mathcal{E}=\left(f_{i}, g_{j k}, r, u_{0 i}, \phi_{0 j k}, \theta_{0}, t_{0 i}, \eta_{0 j k}, q_{0}\right)$.

On the other hand, this system of external actions provokes a thermoelastic state of the body, defined by: $\mathcal{S}=\left(u_{i}, \phi_{j k}, \theta, e_{i j}, v_{i j}, \chi_{i j k}, t_{i j}, \sigma_{i j}, \eta_{i j k}, q_{i}, S\right)$.

\section{Main Results}

As it is known, a Betti result establishes a reciprocity relation between two external action systems and the two corresponding thermoelastic states, which they cause.

Our first theorem refers to a result of this nature.

As such, we shall consider two systems of external loads which act on the thermoelastic dipolar body: $\mathcal{E}^{(v)}=\left(f_{i}^{(v)}, g_{j k}^{(v)}, r^{(v)}, u_{0 i}^{(v)}, \phi_{0 j k}^{(v)}, \theta_{0}^{(v)}, t_{0 i}^{(v)}, \eta_{0 j k}^{(v)}, q_{0}^{(v)}\right), v=1,2$.

Let us denote by $\mathcal{S}^{(v)}$ the two corresponding thermoelastic states, that is: $\mathcal{S}^{(v)}=$ $\left(u_{i}^{(v)}, \phi_{j k}^{(v)}, \theta^{(v)}, e_{i j}^{(v)}, v_{i j}^{(v)}, \chi_{i j k}^{(v)}, t_{i j}^{(v)}, \sigma_{i j}^{(v)}, \eta_{j k}^{(v)}, q_{i}^{(v)}, S^{(v)}\right), v=1,2$.

The product of convolution for the functions $u$ and $v$, which is denoted by $u * v$, is defined by:

$$
\begin{aligned}
& (u * v)(x, t)=\int_{0}^{t} u(x, t-\tau) v(x, \tau) d \tau \\
& (u * \hat{v})(x, t)=\int_{0}^{t} u(x, t-\tau) \frac{\partial v}{\partial \tau}(x, s) d \tau .
\end{aligned}
$$


Theorem 1. Between two external data systems $\mathcal{E}^{(v)},(v=1,2)$ and the two corresponding thermoelastic states $\mathcal{S}^{(v)},(v=1,2)$ there is the following reciprocity relation:

$$
\begin{aligned}
& \int_{B}\left[\left(f_{i}^{(1)} * \hat{u}_{i}^{(2)}-f_{i}^{(2)} * \hat{u}_{i}^{(1)}\right)+\left(g_{j k}^{(1)} * \hat{\phi}_{j k}^{(2)}-g_{j k}^{(2)} * \hat{\phi}_{j k}^{(1)}\right)\right] d V+ \\
& +\int_{S_{1}}\left[\left(\hat{t}_{i j}^{(1)}+\hat{\sigma}_{i j}^{(1)}\right) * u_{0 i}^{(2)}-\left(\hat{t}_{i j}^{(2)}+\hat{\sigma}_{i j}^{(2)}\right) * u_{0 i}^{(1)}\right] n_{j} d A+\int_{S_{1}^{c}}\left[t_{0 i}^{(1)} * \hat{u}_{i}^{(2)}-t_{0 i}^{(2)} * \hat{u}_{i}^{(1)}\right] d A+ \\
& \quad+\int_{S_{2}}\left[\hat{\eta}_{i j k}^{(1)} * \phi_{0 j k}^{(2)}-\hat{\eta}_{i j k}^{(2)} * \phi_{0 j k}^{(1)}\right] n_{i} d A+\int_{S_{2}^{c}}\left[\eta_{0 j k}^{(1)} * \hat{\phi}_{j k}^{(2)}-\eta_{0 j k}^{(2)} * \hat{\phi}_{j k}^{(1)}\right] d A= \\
& \quad=\int_{B} \frac{1}{T_{0}}\left[\left(\theta^{(2)} * r^{(1)}-* \theta^{(1)} * r^{(2)}\right)+\alpha\left(\hat{\theta}^{(2)} * r^{(1)}-\hat{\theta}^{(1)} * r^{(2)}\right)\right] d V+ \\
& \quad+\int_{S_{3}} k_{i j}\left[\left(\theta_{0}^{(2)} * \theta_{, j}^{(1)}-\theta_{0}^{(1)} * \theta_{, j}^{(2)}\right)+\alpha\left(\theta_{0}^{(2)} * \hat{\theta}_{, j}^{(1)}-\theta_{0}^{(1)} * \hat{\theta}_{, j}^{(2)}\right)\right] n_{i} d A+ \\
& \quad+\int_{S_{3}^{c}} \frac{1}{T_{0}}\left[\left(q_{0}^{(2)} * \theta^{(1)}-q_{0}^{(1)} * \theta^{(2)}\right)+\alpha\left(q_{0}^{(2)} * \hat{\theta}^{(1)}-q_{0}^{(1)} * \hat{\theta}^{(2)}\right)\right] d A,
\end{aligned}
$$

where

$$
t_{i}^{(v)}=\left(t_{j i}^{(v)}+\sigma_{j i}^{(v)}\right) n_{j}, \eta_{j k}^{(v)}=\eta_{i j k}^{(v)} n_{i}, q^{(v)}=q_{i}^{(v)} n_{i}, v=1,2 .
$$

Proof. For an original function $f$, we will use the Laplace transform, denoted by $\bar{f}$, which as it is known, is defined through the formula:

$$
\bar{f}(x, s)=\int_{0}^{\infty} f(x, t) e^{-s t} d t .
$$

We will apply the Laplace transform in relation to the variable $t$ to the Equations (3), (5)-(7) and the boundary conditions (10). Thus, if we take into account the initial homogeneous conditions (11), we deduce some equations corresponding to the functions that were transformed. So, we have

- the motion equations:

$$
\begin{aligned}
\left(\bar{t}_{i j}^{(v)}+\bar{\sigma}_{i j}^{(v)}\right)_{, j}+\varrho \bar{f}_{i}^{(v)} & =\rho s^{2} \bar{u}_{i}^{(v)}, \\
\bar{\eta}_{i j k, i}^{(v)}+\bar{\sigma}_{j k}^{(v)}+\varrho \bar{g}_{j k}^{(v)} & =I_{j r} s^{2} \bar{\phi}_{k r}^{(v)} .
\end{aligned}
$$

- the energy equation:

$$
\frac{\varrho}{T_{0}} r^{(v)}+k_{i j} \theta_{, i j}^{(v)}-d s \theta-h s^{2} \theta^{(v)}+a_{i j} s e_{i j}^{(v)}+b_{i j} s v_{i j}^{(v)}+c_{i j k} s \chi_{i j k}^{(v)}+2 b_{i} s \theta_{, i}=0 .
$$

- the constitutive equations:

$$
\begin{gathered}
\bar{t}_{i j}^{(v)}=A_{i j m n} \bar{e}_{m n}^{(v)}+D_{m n i j} \bar{v}_{m n}^{(v)}+F_{m n r i j} \bar{\chi}_{m n r}^{(v)}+a_{i j}(1+\alpha s) \bar{\theta}^{(v)}, \\
\bar{\sigma}_{i j}^{(v)}=D_{i j m n} \bar{e}_{m n}^{(v)}+B_{i j m n} \bar{v}_{m n}^{(v)}+G_{i j m n r} \bar{\chi}_{m n r}^{(v)}+b_{i j}(1+\alpha s) \bar{\theta}^{(v)}, \\
\bar{\eta}_{i j k}^{(v)}=F_{i j k m n} \bar{e}_{m n}^{(v)}+G_{m n i j k} \overline{\bar{v}}_{m n}^{(v)}+C_{i j k m n r} \bar{\chi}_{m n r}^{(v)}+c_{i j k}(1+\alpha s) \bar{\theta}^{(v)}, \\
\bar{S}^{(v)}=-a_{i j} \bar{e}_{i j}^{(v)}-b_{i j} \bar{v}_{i j}^{(v)}-c_{i j k} \bar{\chi}_{i j k}^{(v)}+d(1+\alpha s) \bar{\theta}^{(v)}, \\
\bar{q}_{i}^{(v)}=-T_{0}\left(b_{i} s \bar{\theta}^{(v)}+k_{i j} \bar{\theta}_{i j}^{(v)}\right) .
\end{gathered}
$$


After we apply the Laplace transform, the boundary conditions (10) become:

$$
\begin{aligned}
& \bar{u}_{i}^{(v)}=\bar{u}_{0 i}^{(v)} \text { on } S_{1}, \bar{\phi}_{j k}^{(v)}=\bar{\phi}_{0 j k}^{(v)} \text { on } S_{2}, \bar{\theta}^{(v)}=\bar{\theta}_{0}^{(v)} \text { on } S_{3}, \\
& \bar{t}_{i}^{(v)}=\bar{t}_{0 i}^{(v)} \text { on } S_{1}^{c}, \bar{\eta}_{j k}^{(v)}=\bar{\eta}_{0 j k}^{(v)} \text { on } S_{2}^{c}, \bar{q}^{(v)}=\bar{q}_{0}^{(v)} \text { on } S_{3}^{c} .
\end{aligned}
$$

In the relations (14)-(17) the superscript $v$ takes the values 1 and 2.

Based on equations (14) 1 we can write:

$$
\begin{aligned}
\int_{B} \varrho\left(f_{i}^{(1)} \bar{u}_{i}^{(2)}-f_{i}^{(2)} \bar{u}_{i}^{(1)}\right) d V= & \int_{B}\left[\left(\bar{t}_{i j}^{(2)}+\bar{\sigma}_{i j}^{(2)}\right) \bar{u}_{i}^{(1)}-\left(\bar{t}_{i j}^{(1)}+\bar{\sigma}_{i j}^{(1)}\right) \bar{u}_{i}^{(2)}\right]_{, j} d V+ \\
& +\int_{B}\left[\left(\bar{t}_{i j}^{(1)}+\bar{\sigma}_{i j}^{(1)}\right) \bar{u}_{i, j}^{(2)}-\left(\bar{t}_{i j}^{(2)}+\bar{\sigma}_{i j}^{(2)}\right) \bar{u}_{i, j}^{(1)}\right] d V .
\end{aligned}
$$

In the first integral from right-hand side of (18) we apply the divergence theorem by taking into account the boundary conditions (17). Furthermore, in the second integral from right-hand side of (18) we introduce the constitutive equations (16).

After these transformations, Equation (18) receives the following form:

$$
\begin{aligned}
\int_{B} \varrho & \left(\bar{f}_{i}^{(1)} \bar{u}_{i}^{(2)}-\bar{f}_{i}^{(2)} \bar{u}_{i}^{(1)}\right) d V+\int_{S_{1}}\left[\left(\bar{t}_{i j}^{(1)}+\bar{\sigma}_{i j}^{(1)}\right) \bar{u}_{0 i}^{(2)}-\left(\bar{t}_{i j}^{(2)}+\bar{\sigma}_{i j}^{(2)}\right) \bar{u}_{0 i}^{(1)}\right] n_{j} d A+ \\
& +\int_{S_{1}^{c}}\left[\bar{t}_{0 i}^{(1)} \bar{u}_{i}^{(2)}-\bar{t}_{0 i}^{(2)} \bar{u}_{i}^{(1)}\right] d A=\int_{B}\left(a_{i j}+b_{i j}\right)(1+s \alpha)\left(\bar{\theta}^{(1)} \bar{u}_{i, j}^{(2)}-\bar{\theta}^{(2)} \bar{u}_{i, j}^{(1)}\right) d V .
\end{aligned}
$$

Based on similar considerations, starting from equation $(14)_{2}$, we obtain the following estimate:

$$
\begin{aligned}
& \int_{B} \varrho\left(\bar{g}_{j k}^{(1)} \bar{\phi}_{j k}^{(2)}-\bar{g}_{j k}^{(2)} \bar{\phi}_{j k}^{(1)}\right) d V+\int_{S_{2}}\left(\bar{\eta}_{i j k}^{(1)} \bar{\phi}_{0 j k}^{(2)}-\bar{\eta}_{i j k}^{(1)} \bar{\phi}_{0 j k}^{(2)}\right) n_{i} d A+ \\
& \quad+\int_{S_{2}^{c}}\left(\bar{\eta}_{0 j k}^{(1)} \bar{\phi}_{j k}^{(2)}-\bar{\eta}_{0 j k}^{(2)} \bar{\phi}_{j k}^{(1)}\right) d A=\int_{B} c_{i j k}(1+s \alpha)\left(\bar{\theta}^{(1)} \bar{\phi}_{j k, i}^{(2)}-\bar{\theta}^{(2)} \bar{\phi}_{j k, i}^{(1)}\right) d V .
\end{aligned}
$$

The calculations will be simplified if we will assume that our thermoelastic dipolar body has a center of symmetry at each point, but is otherwise anisotropic. According to [7], this means that $b_{i}=0$, which means a simplification of the energy Equation (15).

So, by using the simplified form (15) of the energy balance, the divergence theorem, the constitutive Equation (6) for $q_{i}$ and the boundary conditions (10), we are led to the following relation:

$$
\begin{gathered}
\int_{B}\left[a_{i j} s\left(\bar{e}_{i j}^{(1)} \bar{\theta}^{(2)}-\bar{e}_{i j}^{(2)} \bar{\theta}^{(1)}\right)+b_{i j} s\left(\bar{v}_{i j}^{(1)} \bar{\theta}^{(2)}-\bar{v}_{i j}^{(2)} \bar{\theta}^{(1)}\right)+c_{i j k} s\left(\bar{\chi}_{i j k}^{(1)} \bar{\theta}^{(2)}-\bar{\chi}_{i j k}^{(2)} \bar{\theta}^{(1)}\right)\right] d V+ \\
+\int_{B} \frac{\varrho}{T_{0}}\left(\bar{\theta}^{(2)} \bar{r}^{(1)}-\bar{\theta}^{(1)} \bar{r}^{(2)}\right) d V=\int_{B} k_{i j}\left(\bar{\theta}^{(1)} \bar{\theta}_{, i j}^{(2)}-\bar{\theta}^{(2)} \bar{\theta}_{, i j}^{(1)}\right) d V= \\
=\int_{B} k_{i j}\left(\bar{\theta}^{(1)} \bar{\theta}_{, j}^{(2)}-\bar{\theta}^{(2)} \bar{\theta}_{, j}^{(1)}\right)_{, i} d V=\int_{S_{3}} k_{i j}\left(\bar{\theta}_{0}^{(1)} \bar{\theta}_{, j}^{(2)}-\bar{\theta}_{0}^{(2)} \bar{\theta}_{, j}^{(1)}\right) n_{i} d A+ \\
+\int_{S_{3}^{c}} \frac{1}{T_{0}}\left(\bar{\theta}^{(2)} \bar{q}_{0}^{(1)}-\bar{\theta}^{(1)} \bar{q}_{0}^{(2)}\right) d A .
\end{gathered}
$$


By combining Equations (19)-(21) we obtain

$$
\begin{array}{r}
s\left[\int_{B} \varrho\left(\bar{f}_{i}^{(1)} \bar{u}_{i}^{(2)}-\bar{f}_{i}^{(2)} \bar{u}_{i}^{(1)}\right) d V+\int_{S_{1}}\left[\left(\bar{t}_{i j}^{(1)}+\bar{\sigma}_{i j}^{(1)}\right) \bar{u}_{0 i}^{(2)}-\left(\bar{t}_{i j}^{(2)}+\bar{\sigma}_{i j}^{(2)}\right) \bar{u}_{0 i}^{(1)}\right] n_{j} d A+\right. \\
+\int_{S_{1}^{c}}\left[\bar{t}_{0 i}^{(1)} \bar{u}_{i}^{(2)}-\bar{t}_{0 i}^{(2)} \bar{u}_{i}^{(1)}\right] d A+\int_{B} \varrho\left(\bar{g}_{j k}^{(1)} \bar{\phi}_{j k}^{(2)}-\bar{g}_{j k}^{(2)} \bar{\phi}_{j k}^{(1)}\right) d V+ \\
\left.\quad+\int_{S_{2}}\left(\bar{\eta}_{i j k}^{(1)} \bar{\phi}_{0 j k}^{(2)}-\bar{\eta}_{i j k}^{(1)} \bar{\phi}_{0 j k}^{(2)}\right) n_{i} d A+\int_{S_{2}^{c}}\left(\bar{\eta}_{0 j k}^{(1)} \bar{\phi}_{j k}^{(2)}-\bar{\eta}_{0 j k}^{(2)} \bar{\phi}_{j k}^{(1)}\right) d A\right]= \\
=(1+s \alpha)\left[\int_{B} \frac{\varrho}{T_{0}}\left(\bar{\theta}^{(2)} \bar{r}^{(1)}-\bar{\theta}^{(1)} \bar{r}^{(2)}\right) d V+\int_{S_{3}} k_{i j}\left(\bar{\theta}_{0}^{(1)} \bar{\theta}_{, j}^{(2)}-\bar{\theta}_{0}^{(2)} \bar{\theta}_{, j}^{(1)}\right) n_{i} d A+\right. \\
\left.+\int_{S_{3}^{c}} \frac{1}{T_{0}}\left(\bar{\theta}^{(2)} \overline{\bar{q}}_{0}^{(1)}-\bar{\theta}^{(1)} \bar{q}_{0}^{(2)}\right) d A\right] .
\end{array}
$$

We now apply in both members of the equality (22) the inverse Laplace transform so that, considering the convolution product, we are led to the reciprocal equality (12). In this way, we have completed the proof of the Theorem 1.

Our second main result is a result of uniqueness with respect to the solution of our mixed initial-boundary value problem $\mathcal{P}$, above defined. For this we need some additional considerations.

For the internal energy $\Psi$ we will consider the following expression:

$$
\begin{aligned}
\Psi= & \frac{1}{2} A_{i j m n} e_{i j} e_{m n}+D_{i j m n} e_{i j} v_{m n}+F_{i j m n r} e_{i j} \chi_{m n r}+ \\
& +\frac{1}{2} B_{i j m n} v_{i j} v_{m n}+G_{i j m n r} v_{i j} \chi_{m n r}+\frac{1}{2} C_{i j k m n r} \chi_{i j k} \chi_{m n r} .
\end{aligned}
$$

As it is known, the generalized free energy function $W$, proposed by Biot, has the expression

$$
W=\Psi-S T_{0}
$$

where $\Psi$ is from (23) and $S$ is the entropy. According to Green and Lindsay [1], the energy function $E$ is given by

$$
E=\Psi-S \Phi,
$$

where the scalar function $\Phi$ is also introduced in [1] and is defined by

$$
\Phi=T_{0}+\theta+\alpha \dot{\theta}+\beta \theta \dot{\theta}+\frac{1}{2} \gamma \dot{\theta}^{2} .
$$

As such, the energy function of Green and Lindsay has the following explicit form:

$$
\begin{aligned}
E=\frac{1}{2} A_{i j m n} e_{i j} e_{m n}+D_{i j m n} e_{i j} v_{m n}+F_{i j m n r} e_{i j} \chi_{m n r}+ \\
+\frac{1}{2} B_{i j m n} v_{i j} v_{m n}+G_{i j m n r} v_{i j} \chi_{m n r}+\frac{1}{2} C_{i j k m n r} \chi_{i j k} \chi_{m n r}+ \\
\quad+\left(a_{i j} e_{i j}+b_{i j} v_{i j}+c_{i j k} \chi_{i j k}\right)(\theta+\alpha \dot{\theta})+\frac{1}{2} \alpha k_{i j} \theta_{, i} \theta_{, j}- \\
-a(\theta+\alpha \dot{\theta})-\frac{1}{2} d \theta^{2}-e \theta \dot{\theta}-\frac{1}{2} f \dot{\theta}^{2}+\alpha b_{i} \dot{\theta} \theta_{, i} .
\end{aligned}
$$


By using the expression of the entropy from (5) ${ }_{4}$, from (24)-(27) we obtain the quadratic form of energy function of Biot, after we consider the initial conditions (11) and we have only retained the quadratic terms:

$$
\begin{aligned}
W=\frac{1}{2} A_{i j m n} e_{i j} e_{m n}+D_{i j m n} e_{i j} v_{m n}+F_{i j m n r} e_{i j} \chi_{m n r}+ \\
+\frac{1}{2} B_{i j m n} v_{i j} v_{m n}+G_{i j m n r} v_{i j} \chi_{m n r}+\frac{1}{2} C_{i j k m n r} \chi_{i j k} \chi_{m n r}+ \\
\quad+\frac{1}{2} \alpha k_{i j} \theta_{, i} \theta_{, j}+\frac{1}{2} d \theta^{2}+h \theta \dot{\theta}+\frac{1}{2} \alpha h \dot{\theta}^{2}-b_{i} \theta \theta_{, i} .
\end{aligned}
$$

Let us denote by $K$ the kinetic energy per unit mass, that is

$$
\mathcal{K}(t)=\frac{1}{2}\left(\varrho \dot{u}_{i}(t) \dot{u}_{i}(t)+I_{j k} \dot{\phi}_{j r}(t) \dot{\phi}_{k r}(t)\right) .
$$

If we use the constitutive equations (4), the motion equations (3), the divergence theorem and denoting by $m$ the mass included in the domain $B$, we obtain

$$
\begin{gathered}
\frac{d}{d t} \int_{m} \mathcal{K}(t) d m=\int_{m}\left\{f_{i} \dot{u}_{i}+g_{j k} \dot{\phi}_{j k}-\frac{1}{T_{0}}\left[A_{i j m n} e_{i j} e_{m n}+D_{i j m n} e_{i j} v_{m n}+\right.\right. \\
+F_{i j m n r} e_{i j} \chi_{m n r}+\frac{1}{2} B_{i j m n} v_{i j} v_{m n}+G_{i j m n r} v_{i j} \chi_{m n r}+ \\
\left.\left.+\frac{1}{2} C_{i j k m n r} \chi_{i j k} \chi_{m n r}+\left(a_{i j} e_{i j}+b_{i j} v_{i j}+c_{i j k} \chi_{i j k}\right)(\theta+\alpha \dot{\theta})\right]\right\} d m+ \\
+\int_{\partial B}\left[\left(t_{i j}+\sigma_{i j}\right) \dot{u}_{j}+\eta_{i j k} \dot{\phi}_{j k}\right] n_{i} d A .
\end{gathered}
$$

Using Equations (6) and (7) and combining Equations (28) and (30), we come to the following equation:

$$
\begin{aligned}
\frac{d}{d t} \int_{m}(\mathcal{K}(t)+ & W(t)) d m=\int_{m}\left[f_{i} \dot{u}_{i}+g_{j k} \dot{\phi}_{j k}+\frac{r}{T_{0}}(\theta+\alpha \dot{\theta})\right] d m+ \\
& +\int_{\partial B}\left[\left(t_{i j}+\sigma_{i j}\right) \dot{u}_{j}+\eta_{i j k} \dot{\phi}_{j k}-\frac{q_{i}}{T_{0}}(\theta+\alpha \dot{\theta})\right] n_{i} d A- \\
& -\int_{\partial B}\left[k_{i j} \theta_{, i} \theta_{, j}+(d \alpha-h) \theta^{2}+b_{i} \theta \theta_{, i}\right] d A .
\end{aligned}
$$

The Equation (31) is the energy equation in the context of Green-Linsday thermoelasticity of bodies with dipolar structure.

As such, by the above estimates, we have actually proved the following theorem.

Theorem 2. In Green-Linsday thermoelasticity of dipolar bodies the energy equation has the form (31).

Remark 1. It is easy to see that the equation of energy balance from Green-Linsday thermoelasticity [1] is a particular case of the Equation (31).

Based on the equality (31) we can prove the uniqueness of solution of the mixed problem $\mathcal{P}$.

Theorem 3.. Assume that the Biot's energy function $W$ is positive definite. Then the mixed problem $\mathcal{P}$ admits at most one solution. 
Proof. Due to the linearity of problem $\mathcal{P}$, the difference of the two supposed solutions is also a solution of problem $\mathcal{P}$, but this difference corresponds to zero loads, that is

$$
f_{i}=g_{j k}=r=0, u_{0 i}=\phi_{0 j k}=t_{0 i}=\eta_{0 j k}=\theta_{0}=q_{0 i}=0
$$

For these zero loads and null boundary conditions, it is easy to see that the energy Equation (31) arrives at the following simplified form:

$$
\frac{d}{d t} \int_{m}(\mathcal{K}(t)+W(t)) d m=-\int_{\partial B}\left[k_{i j} \theta_{, i} \theta_{, j}+(d \alpha-h) \theta^{2}+b_{i} \theta \theta_{, i}\right] d A .
$$

If we consider the initial conditions (11), at $t=0$ we have $\mathcal{K}=W=0$, so that from (32) we deduce

$$
\int_{m}(\mathcal{K}+W) d m \leq 0, \forall t \geq 0
$$

Because we supposed that the function $\mathcal{K}$ is positive definite, it follows that

$$
\mathcal{K}+W=0 \Rightarrow \mathcal{K}=W=0,
$$

for any $t \geq 0$, in which we took into account that $W$ is positive definite.

Clearly, from (33) we can observe that,

$$
u_{i}(t, x)=0, \phi_{j k}(t, x)=0, \theta(t, x)=0, \forall t \geq 0, \forall x \in B,
$$

and this is for the difference of the two solutions.

As such, the proof of the Theorem 3 is completed.

\section{Concluding Remarks}

We established a reciprocal relation which involves thermoelastic processes at different instants. Then we show that this relation can be used to obtain some uniqueness theorems. The reciprocity theorem and also the uniqueness theorems are both derived avoiding the use of the definiteness assumption on the elasticity tensors. Furthermore, we avoided imposing the restriction that the conductivity tensor is positive definite. The new form of energy equation can be considered as an element of novelty of our paper. It is a generalization of the energy equation established in [1]. It can be considered as novelty of our study, as a natural generalization of the previous studies, the transition from bodies with a dipolar structure to the dipolar solids in the context of Green-Lindsay thermoelasticity. Another originality was our tentative to contribute in solving the paradox of the conduction of the heat. Reciprocity relations have both theoretical and practical importance. Theoretically, for instance, these relations can be the basis of some variational principles. So, the solution can be determined as the minimum of the energetic functional. In our future studies we will try to extend such kind of principle to cover the bodies with dipolar structure. The practical importance of reciprocity relations can be explained simply by the fact that a future state of a solid environment can be anticipated, knowing a current state.

Author Contributions: Writing-review and editing, M.M., E.M.C. and N.P. All authors have read and agreed to the published version of the manuscript.

Funding: This work did not receive any funding.

Conflicts of Interest: The authors declare no conflict of interest. 


\section{References}

1. Green, A.E.; Lindsay, K.A. Thermoelasticity. J. Elast. 1972, 2, 1-7. [CrossRef]

2. Green, A.E.; Naghdi, P.M. Re-examination of the basic postulates of thermomechanics. Proc. R. Soc. Lond. A 1991, 432, 1171-1194.

3. Green, A.E.; Naghdi, P.M. On undamped heat wave in elastic solids. J. Therm. Stresses 1992, 15, 253-264. [CrossRef]

4. Green, A.E.; Naghdi, P.M. Thermoelasticity without energy dissipation. J. Elast. 1993, 9, 1-8. [CrossRef]

5. Choudhuri, S.K.R. On a thermoelastic three-phase-lag model. J. Therm. Stresses 2007, 30, 231-238. [CrossRef]

6. Lebon, G. A generalized theory of thermoelasticity. J. Tech. Phys. 1982, 23, 37-46.

7. Svanadze, M.; Scalia, A. Mathematical problems in the coupled linear theory of bone poroelasticity. Comput. Math. Appl. 2013, 66, 1554-1566. [CrossRef]

8. Othman, M.I.A.; Abd-Elaziz, E.M. The effect of thermal loading due to laser pulse in generalized thermoelastic medium with voids in dual phase lag model. J. Therm. Stresses 2015, 38, 1068-1082. [CrossRef]

9. Iesan, D.; Ciarletta, M. Non-Classical Elastic Solids; Longman Scientific and Technical: Harlow, Essex, UK; J. Wiley \& Sons, Inc.: New York, NY, USA, 1993.

10. Mindlin, R.D. Low frequency vibrations of elastic bars. Int. J. Solids Struct. 1976, 12, 27-49. [CrossRef]

11. Green, A.E.; Zerna, W. Theoretical Elasticity, 2nd Revised ed.; Dover Publications: Dover, NH, USA, 2012.

12. Gurtin, M.E.; Fried, E.; Anand, L. The Mechanics and Thermodynamics of Continua; Cambridge University Press: Cambridge, UK, 2010.

13. Marin, M. Lagrange identity method for microstretch thermoelastic materials. J. Math. Anal. Appl. 2010, 363, 275-286. [CrossRef]

14. Othman, M.I.A.; Lotfy, K. The effect of magnetic field and rotation of the 2-D problem of a fiber-reinforced thermoelastic under three theories with influence of gravity. Mech. Mater. 2013, 60, 129-143. [CrossRef]

15. Marin, M.; Nicaise, S. Existence and stability results for thermoelastic dipolar bodies with double porosity. Continuum. Mech. Therm. 2016, 28, 1645-1657. [CrossRef]

16. Marin, M.; Craciun, E.M.; Pop, N. Considerations on mixed initial-boundary value problems for micropolar porous bodies. Dyn. Syst. Appl. 2016, 25, 175-196.

17. Marin, M.; Ellahi, R.; Chirila, A. On solutions of Saint-Venant's problem for elastic dipolar bodies with voids. Carpathian J. Math. 2017, 33, 219-232.

18. Marin, M.; Vlase, S.; Ellahi, R.; Bhatti, M.M. On the partition of energies for the backward in time problem of thermoelastic materials with a dipolar structure. Symmetry 2019, 11, 863. [CrossRef]

19. Othman, M.I.A.; Abbas, I.A. Effect of rotation on plane waves in generalized thermomicrostretch elastic solid: comparison of different theories using finite element method. Can. J. Phys. 2014, 92, 1269-1277. [CrossRef]

20. Singh, A.; Das, S.; Craciun, E.-M. Thermal stress intensity factor for an edge crack in orthotropic composite media. Compos. Part B 2018, 153, 130-136. [CrossRef]

21. Singh, A.; Das, S.; Craciun, E.-M. The effect of thermo-mechanical loading on the edge crack of finite length in an infinite orthotropic strip. Mech. Compos. Mater. 2019, 55, 285-296. [CrossRef]

22. Zenkour, A.M.; Abbas, I.A. Magneto-thermoelastic response of an infinite functionally graded cylinder using the finite element method. J. Vib. Control 2014, 20, 1907-1919. [CrossRef]

23. Pop, N. On the inexact Uzawa methods for saddle point problems arising from contact problem. Bull. Sti. Univ. Baia Mare Math.-Ser. 1999, 15, 45-54.

24. Straughan, B. Heat waves. In Applied Mathematical Sciences; Springer: New York, NY, USA, 2011 ; Volume 177.

25. Eringen, A.C. Microcontinuum Field Theories; Springer: New York, NY, USA, 1999.

26. Chyr, I.A.; Shynkarenko, H.A. Well-Posedness of the Green-Lindsay Variatio-nal Problem of Dynamic Thermoelasticity. J. Math. Sci. 2017, 226, 11-27. [CrossRef]

27. Nieto, M.; Rivera, J.E.M.; Naso, M.G.; Quintanilla, R. Qualitative results for a mixture of Green-Lindsay thermoelastic solids. Chaotic Mod. Simul. 2018, 3, 285-294. 
28. Aouadi, M.; Campo, M.; Copetti, M.I.M.; Fernández, J.R. Analysis of a multidimensional thermoviscoelastic contact problem under the Green-Lindsay theory. J. Comput. Appl. Math. 2019, 1, 224-246. [CrossRef]

29. Kecs, WW.; Toma, A. The quasi-static generalized equation of the vibrations of the elastic bars with discontinuities. Proc. R. Acad. Ser. A 2014, 15, 315-402.

30. Sireteanu, T.; Mitu, A.M.; Giuglea, M.; Solomon, O.; Stefanov, D. Analytical method for fitting the Ramberg-Osgood model to given hysteresis loops. Proc. R. Acad. Ser. A 2014, 15, 35-42.

31. Abbas, I.A.; Zenkour, A.M. The Effect of Rotation and Initial Stress on Thermal Shock Problem for a Fiber-Reinforced Anisotropic Half-Space Using Green-Naghdi Theory. J. Comput. Theor. Nanos 2014, 11, 331-338. [CrossRef]

32. Cimmelli, V.A.; Sellitto, A.; Triani, V. A generalized Coleman-Noll procedure for the exploitation of the entropy principle. Proc. R. Soc. A 2010, 466, 911-925. [CrossRef]

(C) 2020 by the authors. Licensee MDPI, Basel, Switzerland. This article is an open access article distributed under the terms and conditions of the Creative Commons Attribution (CC BY) license (http:/ / creativecommons.org/licenses/by/4.0/). 